\title{
Platelet indicies as an indicators of diabetic nephropathy
}

\section{Martha S. DerisBesada ${ }^{a}$, AbdEIrahman A. Elsayed ${ }^{a}$, Abdallah E. Ali ${ }^{a}$, AbdElqader A. Hashem ${ }^{\mathrm{b}}$}

\author{
${ }^{\text {a }}$ Clinical and chemical pathologydepartement,Qena Faculty of medicine, South \\ Valley University. \\ ${ }^{\mathbf{b}}$ Internal medicine department, Qena Faculty of medicine, South Valley University.
}

\begin{abstract}
:
Background: Diabetic nephropathy is considered one of the most common microvascular complications. Insulin resistance and impaired insulin secretion are very important in the pathogenesis, It can be suggested that increased platelet volume may be an important factor in the enhanced risk of vascular complications.
\end{abstract}

Patients and Method: This study included 50 diabetic patients, 50 diabetic patients with nephropathy \& 50 healthy age and sex-matched subjects as controls. Blood samples were obtained for CBC, Anticoagulated blood (ethylene diamine tetracetic acid) was collected and analyzed in an automated blood cell counter for platelet count and indices (MPV, PDW, P-LCC \& P-LCR), Glucose, HbA1c, Kidney function (creatinine \& urea), Albumin/creatinine ratio \& lipid profile (cholesterol, triglyceride, HDL, LDL \& VLDL) were also done.

Results: MPV, PDW and P-LCR were all significantly higher in diabetic patients compared to the control subjectswith $(\mathrm{P}=0.001,0.05,0.02$ respectively). Among the diabetics, MPV was higher in diabetic patient with nephropathy as compared to those without nephropathy $(\mathrm{P}=0.001)$.

Conclusions:Platelet indices, especially MPV were different between diabetics and controls as well as diabetic nephropathy. Discriminant analysis using MPV and PDW could classify majority of patients with nephropathy.

Keywords: DM, DN, Platelet, Platelet indices.

\section{Introduction:}

DM diagnosedby Glycosylated Haemoglobin(A1C) $\geq 6.5 \%$ or fasting blood glucose(FBG) $\geq 126 \mathrm{mg} / \mathrm{dl}$, fasting should be for at least 8 hours or 2-hour serum glucose $\geq 200 \mathrm{mg} / \mathrm{dl}$ during an oral glucose tolerance test or a random serum glucose $\geq 200 \mathrm{mg} / \mathrm{dl}$ with symptoms of hyperglycemia.Themajority of cases of diabetes can be broadly classified into 2 categories: type I diabetes and type II diabetes although some cases are difficult to classify, gestational diabetes (GDM) refers to glucose intolerance with onset or first recognition during pregnancy(Amed and Oram,2016).

Diabetic nephropathy is considered one of the most common microvascular complications. Insulin resistance and impaired insulin secretion are very important in the pathogenesis.It can be suggested that increased platelet volume may be an important factor in the risk of vascular complications. MPV can be used as a favorable test in the monitoring of diabetic patients in terms of atherosclerosis development.It is proposed that hyperglycemia can increase platelet reactivity by inducing some mechanisms includes the osmotic effect of glucose, non enzymatic protein glycation of the platelet and protein kinase $\mathrm{C}$ activation (Yeboah et al., 2011).

The first symptom of $\mathrm{DN}$ is microalbuminuria that worse towards developing overt proteinuria but only $20 \%$ of patients with the condition progresstowards proteinuric nephropathy.It continues to have a poor prognosis 
withmost patients dying from cardiac disease or progressing to end-stage renal failure, furthermore proteinuria isa marker of vascular endothelial dysfunction and there is a good correlation betweencardiovascular risk and degree of albuminuria(Williams and Pickup, 2004).

Diabetic patients have larger platelets that are more reactive and aggregable and it is thoughtthat platelets may have an important role in the development of atherosclerosis in diabetes. Platelets have insulin binding site and it is assumed that insulin reduces platelet responses against aggregant factors including thrombin, ADP and platelet activating factor. Thus it is found that insulin resistance results in platelet dysfunction(Vinik etal., 2001).

Therefore, increased MPV can generate a pro-coagulant effect and cause thrombotic vascular complications. It can be suggested that there is link between MPV and diabetic vascular complications associated with thrombogenesis, MPV is statistically increased in diabetics with HbA1c levels $\geq 7 \%$ than in diabetics with HbAlc levels < 7\%(Andersson etal.,2012).

Besides, platelet distribution width (PDW) is a parameter that can directly mirror of variability of platelet size. Its high value indicates increased production of larger reticulated platelet and also suggests high activity. Thus numerous studies have focussed on investigating the relationship of MPV and PDW to the development and progression of DN and several studies suggested that there is a positive link, it represents the heterogeneity in PLT morphology thatis clinically related to PLT activation(Kim etal.,2015).

\section{Materials\&Methods:}

- Study design: Aprospective, casecontrol, hospital based study carried out on diabetic patients\& diabetic patients with nephropathy in internal medicine Department in Qenauniversity hospital from June 2019 to February 2020.
- Sample size: 50 Diabetic patients \& 50 diabetic patients with nephropathy in relation to 50 healthy age and sexmatched subjects as controls.

\section{- Target population:}

a. Inclusion criteria: patients diagnosed as diabetes mellitus, able and willing to participate in the study.

b. Exclusion criteria:

Females with $\mathrm{Hb}(<10 \mathrm{~g})$ and

malesHb $(<12 \mathrm{~g})$, patients with coronary artery disease, Pregnant women, Patients on antiplatelet drugs such as aspirin and clopidogrel, Malignancy, Patients with cardiac valve replacement,

Patientswith chronic renal failure on dialysis \& Patients with

thrombocytosis and

thrombocytopenia.

\section{Methodology:}

All of the patients and controls were subjected to blood sampling: $5 \mathrm{ml}$ venous blood sample was collected and divided into 2 tubes; EDTA tube for CBC, HbA1c and plain tubes for random serum glucose, kidney function tests\& lipid profile. Serum samples were obtained by centrifugation of the clotted blood at $3000 \mathrm{rpm}$ for $10 \mathrm{~min}$ at room temperature and second urine specimen was collected in aclean container not older than 4 hours was used.

CBC: using Yumezon H 550 OT analyzerHORIBA ABX SAS, made in france.Platelet indices were obtained from this automated analyzer that include PCT, MPV, PDW, P-LCR, P-LCC.

Glycosylated haemoglobin $(\mathrm{HbA1C})$ that was made on Bio-RAD-10(D-10) Haemoglobin Testing System,made in France.

Random serum glucose, kidney function tests, lipid profile \&Albumin/creatinine ratio were made on Beckman coulter chemistry analyzer AU480, that made in japan.

CBC: was done on automated cell counter.The reference values were: RBCs, 
3.8-6.0x10 $/ \mu 1 . \mathrm{Hb}, \quad 11.5-17.0 \mathrm{~g} / \mathrm{d} 1 . \mathrm{HCT}$, 35.0-52.0\%.MCV, 76.0-100.0 $\mu^{3} . \mathrm{MCH}$, 27.0-34.0pg.MCHC， 32.0-35.0g/dl.WBC, $3.5-10.010^{3} / \mu 1$ and PLT, $150-400 \times 10^{3} / \mu 1$. The platelet indices that done wereMean platelet volume MPV (fl): Analyser calculated measure of thrombocyte volume $=($ plateletcrit $/$ platelet count $\left.\mathrm{x} 10^{9} / \mathrm{I}\right) \times 10^{9}\left(8.0-11.0 \mu \mathrm{m}^{3}\right)$, Plateletcrite (PCT) (\%): Volume occupied by platelet in blood = platelet count $\mathrm{x}$ MPV/10000 (0.150.40), PDW (\%): indicator of volume variability in platelet size $=\mathrm{SD}$ of platelet volume/MPV x 100 (11.0-22.0), P-LCR indicator of the percentage of large $(>12 \mathrm{fl})$ circulating platelet (18.0-50\%).

Random serum glucose, Kidney function tests and Lipid profile:Random serum glucose, $70-140 \mathrm{mg} / \mathrm{dl}$.Creatinine, $0.8-1.3$ $\mathrm{mg} / \mathrm{dl}$.Urea, $15.00-50 \mathrm{mg} / \mathrm{dl}$.Cholesterol till $201.24 \mathrm{mg} / \mathrm{dl}$.Triglyceride, 59.50-164.50 $\mathrm{mg} / \mathrm{dl}$.High density lipoprotein, 29.80$70.82 \mathrm{mg} / \mathrm{dland}$ Low density lipoprotein till130.03mg/dl.

Urine sample:second morning urine specimen was collected in a clean container, non centrifuged not olderthan 4 hours was used and if thesample could not tested immediatelyit was stored at $2-4^{\circ} \mathrm{C}$ and brought at room temperature (15$25^{\circ} \mathrm{C}$ ) for testing. For measurment of creatinine in urine sample, the sample was diluted till 50 times with distalled water and the result was multiplied by 50 and albumin was done in urine then albumin was divided on creatinine to obtain albumin/creatinine ratio (till $2.5 \mathrm{mg} / \mathrm{l}$ in females \& $3.5 \mathrm{mg} / \mathrm{l}$ in males). This test was done on Beckman coulter chemistry analyzer AU 480 that made in Japan.

\section{Statistical analyses}

All analyses were performed with the IBM SPSS 20.0 software. The data were tested for normality usingthe Anderson Darling test and for homogeneity variances prior to further statistical analysis. Categoricalvariables were described by number and percent (N\%) where continuous variables described by mean and standard deviation (Mean, SD). Chisquare used for comparison between categorical variables, whereas independent samples t-test and paired t-test used to compare between continuous variables. Receiver operating characteristic (ROC) curves were constructed to determine the optimal cut off values for platelet indices. The accuracy and the diagnostic performance of platelet indices were compared with the area under the receiver operating curve (AUROC) and the sensitivity, specificity, positive predictive value (PPV), negative predictive value (NPV), were calculated. The diagnosticaccuracy and AUROC were calculated based on platelet indices values as a reference. All calculation is twotailed, $\mathrm{p}<0.05$ was considered statistically significant. Pearson correlation used to detect the association between variables.

\section{Results:}

Demographic data: 50 diabetic patients including(20 males and 30 females) with mean age $48.4 \pm 16.3$ years, 50 diabetic patients with nephropathy including (17 males and 33 females) with mean age $62.3 \pm 14.7$ years, 50 healthy subjectswere selected as a control group withtheir mean age (44.5 \pm 13$)$ years, (23 males and 27 females).

Laboratory data: When compared to control group, diabetic patients with nephropathy had higher levels of MPV, PDW, P-LCR, Creatinine, Urea, Albuminuria, e-GFR, Albumin/creatinine ratio and HDL, Table 1. 
Table 1. laboratory investigation findings in patients and controls:

\begin{tabular}{|l|l|l|l|l|l|l|}
\hline & $\begin{array}{l}\text { Control } \\
\text { group(A) } \\
\mathbf{2 6} \text { cases) }\end{array}$ & $\begin{array}{l}\text { DM group(B) } \\
\mathbf{2 3} \text { cases) }\end{array}$ & $\begin{array}{l}\text { DN group(C) } \\
\mathbf{4 1} \text { cases) }\end{array}$ & $\begin{array}{l}\text { P } \\
\text { value } \\
\text { A\&B }\end{array}$ & $\begin{array}{l}\text { P } \\
\text { value } \\
\text { B\&C }\end{array}$ & $\begin{array}{l}\text { P } \\
\text { value } \\
\text { A\&C }\end{array}$ \\
\hline PLTs & $274.7 \pm 79.6$ & $300.7 \pm 82$ & $279.2 \pm 73.4$ & 0.5 & 0.9 & 0.5 \\
\hline PCT & $0.3 \pm 0.06$ & $0.3 \pm .0 .07$ & $0.3 \pm 0.08$ & 0.9 & 0.9 & 0.9 \\
\hline MPV & $9.4 \pm 1.4$ & $8.1 \pm 0.8$ & $8.4 \pm 1$ & $0.001^{*}$ & 0.4 & $0.001^{*}$ \\
\hline PDW & $15.2 \pm 3.3$ & $12.7 \pm 2.1$ & $13.7 \pm 2.5$ & $0.005^{*}$ & 0.4 & 0.06 \\
\hline P-LCC & $70.4 \pm 20.7$ & $63 \pm 16.8$ & $70.8 \pm 30.5$ & 0.6 & 0.5 & 0.9 \\
\hline P-LCR & $27.5 \pm 9.8$ & $21.1 \pm 6.6$ & $23.3 \pm 8$ & $0.02^{*}$ & 0.5 & 0.1 \\
\hline Glucose & $97.2 \pm 16.9$ & $307.1 \pm 124.5$ & 257.2117 & $0.001^{*}$ & 0.1 & $0.001^{*}$ \\
\hline HBA1c & $5.1 \pm 0.4$ & $9.4 \pm 2.4$ & 8.82 .3 & $0.001^{*}$ & 0.5 & $0.001^{*}$ \\
\hline Creatine & $0.7 \pm 0.2$ & $0.7 \pm 0.2$ & $1.2 \pm 0.4$ & 0.9 & $0.001^{*}$ & $0.001^{*}$ \\
\hline Urea & $22.9 \pm 6.9$ & $24.9 \pm 10.6$ & $42.6 \pm 23.6$ & 0.9 & $0.001^{*}$ & $0.001^{*}$ \\
\hline albuminurea & $14.7 \pm 8.8$ & $89.4 \pm 113.7$ & $311.6 \pm 234.3$ & 0.27 & $0.001^{*}$ & $0.001^{*}$ \\
\hline e-GFR & $131.6 \pm 35.3$ & $123.9 \pm 26$ & $58 \pm 19$ & 0.6 & $0.001^{*}$ & $0.001^{*}$ \\
\hline $\begin{array}{l}\text { Alb/creat } \\
\text { ratio }\end{array}$ & $0.8 \pm 0.4$ & $1.4 \pm 0.6$ & $78.2 \pm 71.6$ & 0.9 & $0.001^{*}$ & $0.001^{*}$ \\
\hline Cholesterol & $172.3 \pm 60.4$ & $167.7 \pm 105.7$ & $177.1 \pm 61.9$ & 0.9 & 0.9 & 0.9 \\
\hline Triglyceride & $142 \pm 101.9$ & $139.2 \pm 89$ & $160.8 \pm 81.7$ & 0.9 & 0.6 & 0.7 \\
\hline HDL & $43.9 \pm 15.4$ & $34.1 \pm 16.8$ & $33.9 \pm 10.7$ & $0.04^{*}$ & 0.9 & $0.01^{*}$ \\
\hline LDL & $108.8 \pm 43.6$ & $111.6 \pm 97.1$ & $113.5 \pm 48.5$ & 0.9 & 0.9 & 0.9 \\
\hline VLDL & $28.5 \pm 20.3$ & $27.8 \pm 17.8$ & $35.1 \pm 24.9$ & 0.9 & 0.4 & 0.5 \\
\hline
\end{tabular}

To differentiate between DM \&Control: ROC curve was estimated to predict significant difference between diabetic patients \&controls in MPV with $(\mathrm{P}$ value $=0.001)$, PDW with $(\mathrm{P}$ value $=0.003) \&$ $\mathrm{P}-\mathrm{LCR}$ with $(\mathrm{P}$ value $=0.008)$. Figure 1.

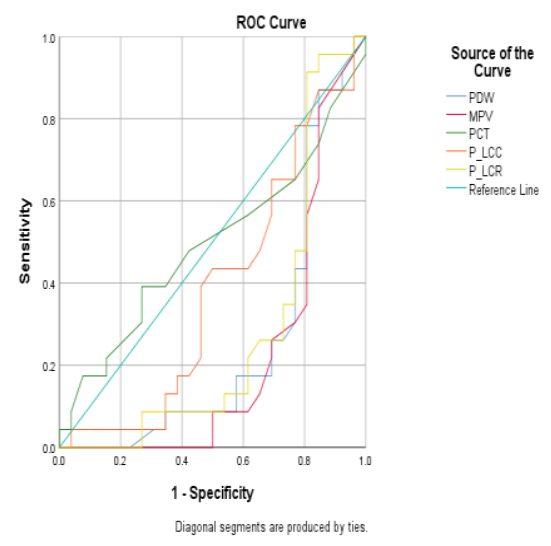

Figure 1.ROC curve between DM \& Control
To differentiate between diabetic patients with nephropathy \&control:

In ROC curve there was significant difference between diabetic patients with nephropathy \& control group in MPV with (P value=0.004), PDW with $(\mathrm{P}$ value $=0.036)$.

Figure 2.

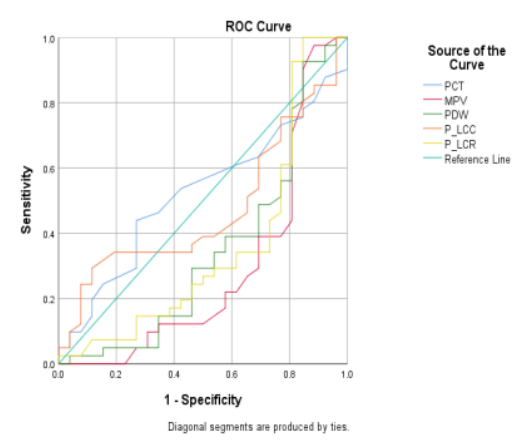

Figure 2.ROC curve between diabetic patients with nephropathy \& Control. 


\begin{abstract}
Discussion
$\mathrm{DM}$ is a 'prothrombotic state' associated with accelerated atherosclerosisand inflammation. Patients with diabetes, particularly those with type II DM, have been shown to exhibit increasing platelet reactivity. This has been attributed to both insulin resistance and insulin deficiency. Insulin has been shown to antagonize the effect of platelet agonists like collagen, adenosine diphosphate, epinephrine and platelet activating factor (Westerbacka etal.,2002).
\end{abstract}

Hyperglycemia contributes to heightened platelet reactivity directly as well as through glycationof platelet proteins. In addition, hyper triglyceridemia also increases platelet reactivity, enhanced platelet aggregation has been implicated in the development of micro- and macrovascular disease in patients with DM, astudy involving uncomplicated insulin-dependent diabetic patients concluded that platelet function abnormalities were absent in diabetic patients without clinical evidence of vascular disease(Hendra etal., 1988). There have been few studies in the available literature on platelet indices in patients with DM. An increase in MPV has been documented in patients with metabolic syndrome, stroke and DM. Increased MPV has also been shown to be an independent risk factor for myocardial infarction, cerebral ischemia and transient ischemic attack. In earlier studies, MPV was found to be significantly higher in diabetic patients and it was hypothesized that platelets with altered morphology are likely to be associated with increased risk of vascular complications in diabetes(Hekimsoy etal.,2004).

Larger platelets contain more dense granules and hence are more potent and thrombogenic.In the present study
MPV was significantly higher in diabetic patients compared to controls $(\mathrm{P}=0.001)$. In addition, other platelet indices (PDW and P-LCR) were also higher in diabetic subjects as compared to controls and also diabetic patients with nephropathy as compared to controls $(\mathrm{P}=0.001$ and 0.001 , respectively). The effect of platelet count on these platelet indices was ruled out, since platelet counts were similar in both three groups(Chu et al., 2010).

MPV was not significantly different between diabetic patients and diabetic patients with nephropathy in our study. However, an interesting finding in the present study was the highly significant difference in PDW in diabetic patients with complications compared to control group.

These results are encouraging in that these factors may act as indicators of microvascular complications(diabetic nephropathy). This is particularly important because:

(1) These indices are conveniently obtained from automated cell counters; (2) Microvascular complications of diabetes are significant causes of morbidity in diabetics and are usually detected quite late in the course of disease.

(3) Early indication of the presence of one of the complications would go a long way in reducing the morbidity and health care costs in patients with diabetes(Schneider,2015).

In our study, the MPV was significantly higher in the diabetic group than the non diabetic controls which was similar to the studies done by other researchers, unlike in an earlier study by Akinsegunetal that showed lower MPV in diabetic cases compared to the controls with no statistical significant difference. In addition, other platelet indice PDW was also significantly higher in diabetic subjects compared to controls 
$(\mathrm{P}=0.005)$. Similar results were noted in other studies done by Demirtasetal, Jabeenetal. and Dalamagaetal with significantly higher PDW levels among diabetic cases(Demirtas etal.,2004).

The P-LCR is not often quoted in literature, probably because it is relatively a new platelet volume parameter. It is generated by only a few machines, with the Yumezenanalyser being one of them. my study concluded that P-LCR was significantly higher ( $P$ value 0.02 ) in diabeticcases. This is in agreement with the studies done by Jindal etal and Ashraf etal which concluded significantly higher P-LCR in diabetes compared to non diabetics(Ashraf etal.,2017).

In agreement with studies done by Kodiateetal and Mowafyetal, my study showed non statistically significant higher MPV in diabetic patients and diabetic patients with nephropathy. Unlike in the study by Papanasetal and Demirtasetal, concluded significantly higher MPV in diabetics with complications than without complications in their study as a role for the increased platelet activity in the pathogenesis of vascular complications. observed a statistically significant higher PDW and P-LCR in diabetics with complications than without complications. This was in agreement with Jindal etal(Jindal etal.,2011).

In my study, MPV, PDW and P-LCR were significantly higher in diabetics with HbA1c levels $\geq 6.5 \%$ than in diabetics with HbA1c levels $\leq 6.5 \%$. This is in agreement with the studies conducted by Kodiatteetal, Ozder and Eker, Ulutasetal and Demirtasetal.
There were higher number of diabetics with HbA1c levels $\geq 6.5 \%$ which is similar to the observation in the study done by Kodiatteetal. This might have been due to poor dietary practices and lack of knowledge regarding the diet and exercise regimens that was obsorved in diabetes. Ozder and Eker also concluded that as glycemic control improves, HbA1c and MPV tends to decrease. Therefore, it may be concluded that glycemic control improves platelet activity and function and maydelaypossible diabetic vascularcomplications $(\boldsymbol{O}$ zder\&Eker,20 14).

Conclusion:Ourstudy suggest that increased platelet volume indices and larger platelets contributes to the prothrombotic state in diabetes mellitus. Because larger platelets are hemostatically more active, therefore its presence probably is a risk factor fordeveloping diabetic vascular complications. larger platelets can be easily identified during routine hematological analysis as MPV, PDW and P-LCR that are generated as byproduct of the automated blood counts. Hence, MPV, PDW and PLCR would be a useful prognostic marker of vascular complications in diabetes.

However, the increased MPV, PDW and P-LCR as the cause or the end result of vascular complications needs to be further explored. Thus, platelet indices MPV, PDW and P-LCR provides an important, simple, effortless and cost effective tool which can be useful in predicting an impending thrombotic state and vascular complications of diabetes. 
References:

Amed S and Oram R, (2016). Maturity-Onset Diabetes of the Young (MODY): Making the right diagnosis to optimize treatment;40:449-54.

Yeboah J, Bertoni A. G, Herrington D. M, Post W. S and Burke G. L, (2011). Impaired fasting glucose and the risk of incident diabetes mellitus and cardiovascular events in an adult population: MESA (Multi- Ethnic Study of Atherosclerosis) J AM CollCardiol;58:140-146.

Williams G and Pickup JC,(2004). The handbook of diabetes, 3rd edn.Oxford Blackwell Publishing.

Vinik A. I, Erbas T, Park T. S, Nolan $R$ andPittenger $G$. $L$, (2001). Platelet dysfunction in type IIdiabetes. DiabetesCare ;24:14761485.

Andersson C, Van Gaal L, Caterson I. D, Weeke P, James W. P, Coutinho W etal., (2010). Relationship between HbA1C levels and risk of cardiovascular adverse outcomes and all-cause mortality in overweight and obese cardiovascular high-risk women and men with type II diabetes. Diabetologia;55:2348-2355.

Kim D. S, Lee J, Kim S. H, Kim S. $M$ and Lee M. G, (2015). Mean platelet volumeis elevated in patientswith psoriasis vulgaris. YonseiMed J;56:712-8.

Westerbacka J, Yki-Jarvinen H, Turpeinen A, Rissanen A, Vehkavaara S, Syrjala M, etal., (2002). Inhibition ofplatelet-collagen interaction: an in vivo action ofinsulin abolished by insulinresistance in obesity.

ArterosclerThrombVascBiol ;22:167-72.

Hendra T. J, Oughton J, Smith C. C, Betteridge D. J andYudkin J. S, (1988). Platelet function in uncomplicated insulin-dependent diabetes patients at rest andfollowing exercise.Diabetes Med;5:469-73.

Hekimsoy Z, Payzin B, Ornek T and Kandogan G,(2004). Mean platelet volume in Type II diabetic patients. JDiabetesComplications;18:1 73-176.

ChuS.G, Becker R.C, Berger P.B, Bhatt D.L, Eikelboom J. W, Konkle B etal.,(2010). Mean platelet volume as apredicator of cardiovascular risk: asystematic review of platelet dysfunction and metaanalysis.JThromb.Haemost.8;148-156 10.1111/j.1538-7836.2009.03584.

Schneider D. J, Shimizu I, Makino H, Imagawa A, Iwahashi $H$, Uchigata Y etal., (2011). Factorscontributing to increased platelet"Clinical EvidenceHandbook: Diabetic Nephropathy: Preventing Progression-American Family Physician". 83 (6): 732

Retrieved 2015-06-27.

Demirtas L, Degirmenci H, Akbas E, Ozcicek A, Timuroglu A,Gure etal., (2004). World Health Organization Guideline.Type I diabetes: recentdevelopments. BMJ; 328:750754.

Ashraf S, Ranjan R, Singh S, Singh $H$, Kudesia $M$ and Sharma $R$, (2017). Diabetes disease burden by platelet indicesas possible biomarkers in evaluationof vascular risks in grading diabetes initial mellitus: correlation indices withhematopoietic 
and biochemicalbiomarkers in diabetes mellitus. Open J Biochem 1-15 (in press).

Jindal S, Gupta S, Gupta R, Kakkar A, Singh H. V, Gupta Ketal., (2011). Platelet indices in diabetes mellitus: indicators of diabetic microvascular complication Hematology16:86-90.

Ozder A, Eker H,P. Blair and R. F laumenhaft, (2014). Investigation of mean platelet volume in "Platelet alpha-granules:basic biology and clinical correlates," Blood Reviews,vol. 23, pp. 17189,2009. 